\title{
Rapid induction of orthotopic hepatocellular carcinoma in immune-competent rats by non-invasive ultrasound-guided cells implantation
}

\author{
Hoi-Hung Chan ${ }^{1,2,7 \dagger}$, Tian-Huei Chu ${ }^{5 \dagger}$, Hsin-Fan Chien ${ }^{5}$, Cheuk-Kwan Sun ${ }^{6}$, E-Ming Wang ${ }^{1,2}$, Huay-Ben Pan ${ }^{3,4}$,
} Hsiao-Mei Kuo ${ }^{1}$, Tsung-Hui Hu ${ }^{8}$, Kwok-Hung Lai ${ }^{2,7}$, Jiin-Tsuey Cheng ${ }^{1 *}$, Ming-Hong Tai ${ }^{3,5^{*}}$

\begin{abstract}
Background: The fact that prognoses remain poor in patients with advanced hepatocellular carcinoma highlights the demand for suitable animal models to facilitate the development of anti-cancer medications. This study employed a relatively non-invasive approach to establish an orthotopic hepatocellular carcinoma model in immune-competent rats. This was done by ultrasound-guided implantation of cancer cells and the model was used to evaluate the therapeutic efficacy of short-term and low-dose epirubicin chemotherapy.
\end{abstract}

Methods: Rat Novikoff hepatoma cells were injected percutaneously into the liver lobes of Sprague-Dawley rats under the guidance of high resolution ultrasound. The implantation rate and the correlation between dissected and ultrasound-measured tumor sizes were evaluated. A similar induction procedure was performed by means of laparotomy in a different group of rats. Pairs of tumor measurement were compared by ultrasound and computerized tomography scan. Rats with a successful establishment of the tumor were divided into the treatment (7-day low-dose epirubicin) group and the control group. The tumor sizes were non-invasively monitored by the same ultrasound machine. Blood and tumor tissues from tumor-bearing rats were examined by biochemical and histological analysis respectively.

Results: Ultrasound-guided implantation of Novikoff hepatoma cells led to the formation of orthotopic hepatocellular carcinoma in 60.4\% (55/91) of the Sprague-Dawley rats. Moreover, tumor sizes measured by ultrasound significantly correlated with those measured by calipers after sacrificing the animals $(P<0.00001)$. The rate of tumor induction by ultrasound-guided implantation was comparable to that of laparotomy $(55 / 91,60.4 \%$ vs. $39 / 52,75 \%)$ and no significant difference in sizes of tumor was noted between the two groups. There was a significant correlation in tumor size measurement by ultrasound and computerized tomography scan. In tumorbearing rats, short-term and low-dose epirubicin chemotherapy caused a significant reduction in tumor growth, and was found to be associated with enhanced apoptosis and attenuated proliferation as well as a decrease in the microvessel density in tumors.

Conclusions: Ultrasound-guided implantation of Novikoff hepatoma cells is an effective means of establishing orthotopic hepatocellular carcinoma in Sprague-Dawley rats. Short-term and low-dose epirubicin chemotherapy had perturbed tumor progression by inducing apoptosis and neovascularization blockade.

\footnotetext{
* Correspondence: tusya@mail.nsysu.edu.tw; minghongtai@gmail.com

† Contributed equally

'Department of Biological Sciences, National Sun Yat-sen University, 70 Lien-

Hai Road, Kaohsiung 80424, Taiwan

${ }^{3}$ Department of Education and Research, Kaohsiung Veterans General

Hospital, 386 Ta-Chung 1st Road, Kaohsiung 81362, Taiwan
} 


\section{Background}

Hepatocellular carcinoma (HCC) is the most common primary malignancy of the liver (70-85\%). It is also one of the most frequent malignancies worldwide, particularly in Asia and Africa. The incidence is still rising in some countries such as Central Europe, North America and Oceania for unknown reasons [1]. Unfortunately, most of the HCC patients have non-specific symptoms [2] and will probably miss the chance of receiving curative treatment. Ultrasound (with or without contrast agents) is sensitive in detecting small HCCs while new generation computerized tomography (CT) with spiral and triphasic scanners can improve the specificity in differentiating $\mathrm{HCC}$ from other kinds of liver tumors. Serum $\alpha$-fetoprotein (AFP) is probably the most frequently used tumor marker for the diagnosis of HCC. However, the sensitivity and specificity of AFP need further validation such as exploration of its subtypes. Routine use of percutaneous needle biopsy of $\mathrm{HCC}$ is controversial because of the risk of needletrack seeding and is better reserved for situations where definite histological diagnosis is mandatory $[3,4]$. Although tumor resection and liver transplantation are currently the mainstays of curative therapies for HCC, only $10-15 \%$ of newly diagnosed patients in Asia have resectable tumors. Local therapies such as radiofrequency ablation and alcohol injection are alternatives for small tumors and patients unsuitable for surgical intervention with comparable success rates. Transarterial chemoembolisation (TACE) is recommended for selected cases of locally advanced large unresectable tumors with good liver functional reserve and no vascular involvement [5].

Since prognoses are dismal for advanced or metastatic tumors [6], the development of a suitable model for testing new treatment modalities for HCC is urgently required. Screening of drug candidates for HCC is usually performed using xenografted $\mathrm{HCC}$ in immunedeficient mice such as nude or severe combined immunodeficiency (SCID) mice. In such xenografted models, tumors are relatively vulnerable because they are not grown in vascularized livers. In addition, those studies fail to delineate the efficacy of therapeutic agents in animals with intact immune systems. In order to develop clinically applicable intervention strategies for HCC, it is essential to create an immune-competent animal model bearing orthotopic HCC.

To create animal models with orthotopic HCC, the implantation of hepatoma cells through laparotomy into syngeneic or immune-deficient animals have been employed in previous studies $[7,8]$. However, it is rather time-consuming and traumatic. Besides, the experimental animals often suffer from adverse events such as bleeding, infection, and tumor adhesion to the tissues and organs. Conditions are even more complicated when repeated monitoring of the tumor status is needed. Percutaneous therapies such as radiofrequency ablation with the guidance of real-time Ultrasound (US) have been widely used for the treatment of HCC with high efficacy and safety [9]. An US machine can also be used as a tool for tumor implantation and measuring the subsequent changes of the created tumors [8]. However, due to the small size of rat livers compared with those of humans, it is a prerequisite to have a high resolution US machine as well as a high frequency probe for precise tumor measurement and US-guided injection. In the current study, therefore, we investigated the feasibility and efficacy of creating orthotopic HCC (transplantable liver cancer) in a rodent model by US-guided implantation. We employed Novikoff hepatoma (N1-S1) cells, which were derived from Sprague-Dawley (SD) rats administered with $\mathrm{N}$-2-fluorenylphthalamic acid (FPA), for US-guided implantation into the liver lobes of SD rats.

Epirubicin, which is similar to doxorubicin as a derivative of anthracycline, can inhibit topoiosomerase II- $\alpha$ (TOP2A) enzyme through preventing the cleavage of supercoiled DNA and blocking DNA transcription and replication. It has been used both alone [10] and in combination with other anticancer agents $[11,12]$ in the treatment of advanced HCC. Studies have shown that regimens containing either epirubicin or doxorubicin resulted in similar response rates and survival. However, epirubicin caused less bone marrow suppression and cardiotoxicity than doxorubicin [13]. Metronomic dosing, a new concept of chemotherapy first described by Browder et al., [14] and Klement et al., [15] is the use of low-dose cytotoxic drugs, either by continuous infusion or frequent administration without extended resting periods in the treatment of malignancy. It is not as toxic as the traditional maximum tolerated dose (MTD) of chemotherapy and better accepted by patients. It also has the additional benefit of targeting the tumor endothelium instead of tumor cells resulting in an antiangiogenic effect [16].

In the current study, we first evaluated the feasibility of using US-guided implantation of N1-S1 cells to generate orthotopic $\mathrm{HCC}$ in rats and the reliability of using the same machine to monitor tumor progression within the rats. Subsequently, rats bearing established HCC were treated with short-term (7-day) and low-dose epirubicin chemotherapy. We aimed at evaluating the therapeutic efficacy of this method and the related mechanism underlying the treatment in this novel HCC model. 


\section{Methods \\ Cell cultures}

Rat Novikoff hepatoma (N1-S1) cells were cultured in the RPMI 1640 medium (Life Technologies, Rockville, MD) containing $100 \mathrm{U} / \mathrm{mL}$ penicillin (Hyclone, BioCheck Laboratories Ltd., USA), $100 \mu \mathrm{g} / \mathrm{mL}$ streptomycin (Hyclone, Bio-Check Laboratories Ltd., USA), 5\% fetal calf serum (GIBCO BRL, Rockville, MD) and $2 \mathrm{mmol} / \mathrm{L}$ glutamine (Hyclone, Bio-Check Laboratories Ltd., USA) under humidified conditions in $95 \%$ air and $5 \% \mathrm{CO}_{2}$ at $37^{\circ} \mathrm{C}$. A rat hepatocyte cell line (Clone-9) derived from normal Sprague-Dawley rat liver was purchased from Bioresource Collection and Research Center ( no. 60201; Hsin-Chu, Taiwan).

\section{Animal studies}

All experimental procedures were reviewed and approved by the Institutional Animal Care and Use Committee before the study began, and we ensured that all animals received humane care and that study protocols complied with the institution's guidelines (see Additional file 1). Male Sprague-Dawley (SD; $150 \pm 50$ g) rats purchased from the National Animal Center (Taipei, Taiwan) were used in this study. Guidelines for pain experiments on conscious animals were adhered to throughout the experiments. The animals were housed two per cage, in [beta]-chip lined metal cages in a central animal care facility with a 12-hour light and 12-hour dark cycle. They were fed with rat chow and water ad libitum. After anesthetization, N1-S1 cells $(5 \times$ $10^{6}$ cells suspended in $100 \mu \mathrm{l}$ of RPMI1640) were injected percutaneously into the liver parenchyma of the SD rats using a 29-gauge syringe under the guidance of real-time US with a high frequency linear array transducer operating at 7-14 $\mathrm{MHz}$ (GE Logiq $9^{\circ}$ Ultrasound System). Similar induction procedure was performed by means of laparotomy in a different group of rats.

Ten days after induction, the animals were anesthetized and tumor sizes were measured by US in two perpendicular diameters and expressed as the mean of the two measurements [8]. Subsequently, the rats were sacrificed and the HCC sizes were measured by calipers. Alternatively, tumor volume was estimated by the formula: volume $=(\text { shortest diameter })^{2} \times($ longest diameter $\times 0.5$ [17]. Plasma samples were collected from tumor-bearing rats and subjected to biochemical analysis including glutamic oxaloacetic transaminase (GOT) and glutamic pyruvic transaminase (GPT) activities using an automatic biochemical analyzer (DAX96, Bayer Corp. Diagnostic, Milan, Italy). Fifteen pairs of tumor measurement were performed using US and functional (perfusion) computerized tomography (CT) scan as previously described by Kan Z [18], while the results of biochemical measurements were plotted and compared by Pearson's correlation.

\section{Short-term (7-day) and low-dose epirubicin chemotherapy} for treatment of HCC in SD rats

After successful implantation of orthotopic HCC in the $10^{\text {th }}$ day, SD rats with comparable sizes of tumor were equally divided into the treatment $(n=11)$ group and the control $(\mathrm{n}=11)$ group. Low-dose epirubicin at 0.3 $\mathrm{mg} /$ day/rat was injected through the tail vein in the treatment group for seven days (the risk of congestive heart failure might occur at cumulative doses greater than $900 \mathrm{mg} \mathrm{m}^{-2}$, about $300 \mathrm{mg} \mathrm{kg}^{-1}$ ) $[19,20]$. After completion of treatment, tumor sizes measured by US were compared between groups and with those before treatment. Moreover, actual tumor sizes were obtained by caliper measurement after sacrificing the animals and the tumors were also weighted. Peripheral blood was drawn from the tail veins of the rats after completion of treatment to check the white and red blood cells as well as the platelet counts.

\section{Western blot analysis}

The protein extracts were isolated using RIPA buffer (150 mM NaCl, $50 \mathrm{mM}$ HEPES pH 7, 1\% Triton X-100, $10 \%$ glycerol, $1.5 \mathrm{mM} \mathrm{MgCl}$, 1 mM EGTA) containing a protease inhibitor (Roche Applied Science; Indianapolis, IN). After separation in $12.5 \%$ SDS-PAGE, protein samples were transferred onto polyvinylidene fluoride (PVDF) membrane using blotting apparatus. The membrane was blocked with 5\% milk in TBS-T for $1 \mathrm{~h}$. Then, it was incubated with TOP2A antibody (1:500, cell-signaling) for $1 \mathrm{~h}$ at room temperature. After incubation with HRP-conjugated secondary antibody (1:5000 dilutions in $5 \%$ milk) for 60 minutes, the signals on membrane were detected using ECL-plus luminol solution (Pharmacia; Piscataway, NJ) and exposed to X-ray film for autoradiogram.

\section{Immunohistochemical analysis}

The paraffin-embedded tissue blocks were sectioned into 3-millimeter slices and mounted on poly-L-lysinecoated slides. After deparaffinization, the slides were blocked with 3\% hydrogen peroxide for 10 minutes and subjected to antigen retrieval by microwave in $10 \mathrm{mM}$ citrate buffer for 15 minutes. TOP2A (1:50, cellsignaling), Ki-67 antibodies (1:100 dilution, Dako, Denmark) and PECAM-1 (platelet endothelial cell adhesion molecule-1, 1:50 dilution; Santa Cruz Biotechnology Inc.) were applied onto the sections, which were then incubated at room temperature for 60 minutes followed by repeated washing with phosphate-buffered saline (PBS). Horseradish peroxidase/Fab polymer conjugate 
(Polymer Detection System, Zymed, USA) was then applied to the sections and the sections were incubated for 30 minutes. After rinsing with PBS, the sections were incubated with peroxidase substrate diaminobenzidine (1:20 dilution, Zymed) for 5 minutes. Thereafter, the sections were counterstained with Gill's hematoxylin for 20 seconds, dehydrated with serial ethyl alcohol, cleared with xylene, and finally mounted. For comparison, results of the percentage of Ki-67-positive staining were counted under low power fields while PECAM-1 was presented as the amount of positive staining under high power fields.

The terminal deoxynucleotidyl transferase-mediated dUTP nick end-labeling (TUNEL) staining

The TUNEL assay was used to detect DNA fragmentation. Briefly, the hepatoma sections on slides were deparaffinized, and then washed with PBS. TUNEL analysis was performed using the in situ Cell Death Detection Kit Fluorescein (Roche Molecular Biochemicals; Indianapolis, IN) according to the manufacturer's protocol. TUNEL-positive cells were visualized by immunofluorescent microscopy and counted using a $20 x$ objective. TUNEL-positive cells containing FITC were identified by co-localization with 4,6-diamidino-2phenylindole (DAPI) staining and by morphology. More than 100 cells were counted for each variable per experiment. The slides were viewed under a fluorescence microscope with green fluorescence set at $520 \mathrm{~nm}$. The cells stained green indicated apoptotic cells. For comparison, the amount of positive staining was counted under low power fields.

\section{Statistical analysis}

The induction rate of HCC by the US-guided method was presented as the percentage of rats with successful tumor induction divided by the total number of rats enrolled. The tumor sizes were expressed as mean \pm standard deviation. Pearson's correlation was applied to compare the results of US with the corresponding measurements. Continuous variables were compared with the Student's $t$ test. A $P<0.05$ on two-tailed testing was considered significant.

\section{Results}

Ultrasound-guided implantation of N1-S1 cells effectively induced orthotopic HCC in SD rats

To generate orthotopic $\mathrm{HCC}$ in immune-competent rats, N1-S1 cells were implanted into the liver lobes of the anesthetized SD rats under the guidance of high resolution US on day 1 (Figure 1a). After 10 days, US revealed prominent HCC in $60.4 \%$ (55/91) of animals with an average size of $16.23 \mathrm{~mm}$ (Figure 1b). The presence of HCC was further confirmed after sacrificing animals for caliper measurement (Figure 1c). The rate of tumor induction by US was comparable to that of laparotomy (55/91, $60.4 \%$ vs. $39 / 52,75 \%$ ) and no significant difference in the sizes of tumor was found between the two groups (Number of rats: US vs. laparotomy: 55 vs. $39, P=0.6759$, Figure 1d). Indeed, it took much less time to perform ultrasound implantation. Besides, the animals recovered better and were free of adhesive tumor nodules at injection sites. Therefore, ultrasoundguided implantation of N1-S1 cells into liver lobes is an effective means of inducing orthotopic HCC in SD rats.

The ultrasound-measured HCC size highly correlated with the caliper measurement, plasma GOT level, and CTmeasured tumor size

To evaluate the accuracy of US measurement, we first compared the US-measured HCC sizes with the caliper measurement immediately after sacrificing the animals. Arithmetic mean of tumor diameters were calculated from width and length or alternatively, tumor volume was estimated by the formula: volume $=$ (shortest diameter $)^{2} \times$ (longest diameter $) \times 0.5$. By using arithmetic mean, the HCC sizes measured by US $(16.23 \pm 12.58$ $\mathrm{mm} ; \mathrm{n}=55)$ were similar to those of caliper measurement $(18.63 \pm 12.95 \mathrm{~mm} ; \mathrm{n}=55)$. Moreover, there was an excellent correlation between US-measured and caliper-measured HCC sizes (Pearson's correlation coefficient $=0.8697, \mathrm{n}=55,{ }^{* * * *} P<0.0001$, Figure 2a).

The biochemical parameters in blood samples from HCC-bearing rats were determined to investigate the relationship between US-measured HCC sizes and plasma GOT/GPT levels. Interestingly, the US-measured HCC sizes significantly correlated with plasma GOT level (Pearson's correlation coefficient $=0.6484, \mathrm{n}=71$, ${ }^{* * * *} P<$ 0.0001; Figure 2b), but not with GPT (data not shown).

As CT scan is a fast and reliable means for acquiring two-dimensional tumor images, a portion of HCCbearing rats were subjected to $\mathrm{CT}$ scan examination immediately after US diagnosis (Figure 2c). There was a significant correlation in the tumor size measured (arithmetic mean) by US and CT scan (Pearson's correlation coefficient $=0.687, \mathrm{n}=15,{ }^{*} P<0.05$; Figure $2 \mathrm{~d}$ ).

In addition, similar results were obtained by comparing the tumor volumes (US vs. caliper: $\mathrm{n}=55$, correlation coefficient $=0.7988,{ }^{* * *} P<0.0001$; US vs. CT: $\mathrm{n}=$ 15 , correlation coefficient $=0.9099,{ }^{* * *} P<0.0001$ ). These findings strongly supported the fidelity of US-based monitoring of $\mathrm{HCC}$ progression in rats.

\section{Short-term and low-dose epirubicin chemotherapy led to stable disease in rats bearing established $\mathrm{HCC}$}

Subsequently, rats bearing established HCC were subjected to short-term (7-day) and low-dose epirubicin therapy to validate this animal model for drug screening 

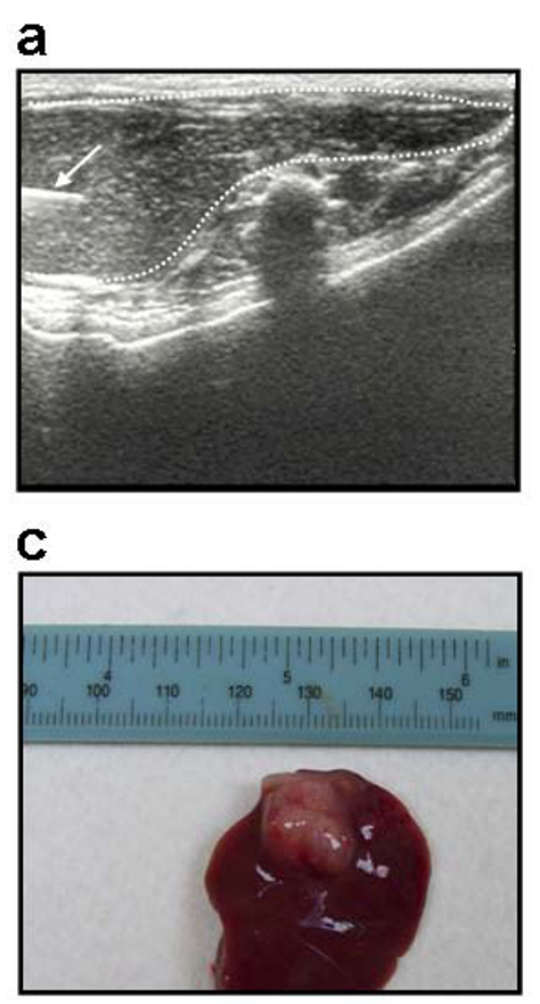

Figure 1 Ultrasound (US) -guided implantation of orthotopic HCC. (a) US-guided implantation on day 1. The needle track was shown on the left of the picture (arrow). (b) US follow-up showing tumor growth on day 10. (c) The appearance of HCC after the sacrifice of rats. (d) No significant difference in sizes of tumor formation with regard to the methods of induction by US and laparotomy were found (Number of rats: US vs. laparotomy: 55 vs. $39, P=0.6759)$.

(Figure 3a). When tumors were successfully established on day 10 , the animals were divided into the treatment (7-day low-dose epirubicin) group and the control group. After completion of treatment, ultrasound monitoring revealed that the tumor burden in the control group continued to increase $\left({ }^{* *} P<0.01\right.$; Figure $\left.3 \mathrm{~b}\right)$, whereas the tumors in rats treated with epirubicin showed no significant increment $(P=0.1737$; Figure $3 \mathrm{~b})$. After sacrificing the animals, it was found that the tumor sizes of the control group were significantly larger than those of the epirubicin group (" $P$ $<0.05$; Figure 3c) while the tumor weights exhibited a similar trend but showed no statistical significance $(P=$ 0.0906; Figure 3d). On the other hand, epirubicin treatment caused significant bone marrow suppression relative to the control group in terms of pancytopenia (Figures 4a, $4 \mathrm{~b}$ and $4 \mathrm{c})$.

\section{Short-term and low-dose epirubicin chemotherapy induced apoptosis and neovascularization blockade in HCC}

Western blotting demonstrated abundant TOP2A enzyme expressions in N1-S1 cells using protein extracts from cancer cells and normal Clone 9 hepatocytes b

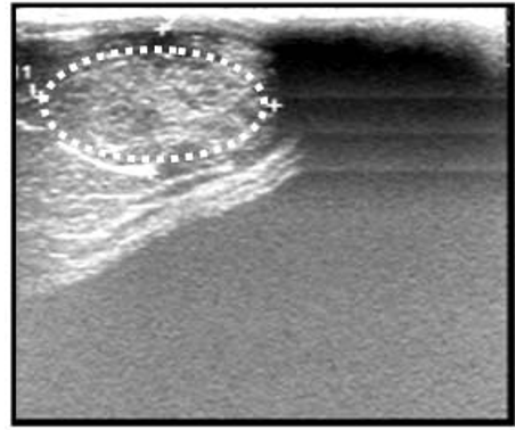

d

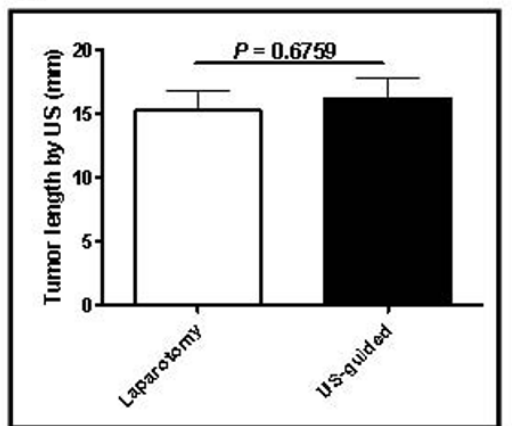

(Figure 5a). Consistent results were also obtained from immunohistochemical studies in tumor specimens ( $\mathrm{T}$ : tumor vs. N: non-tumor parts; Figures $5 \mathrm{~b}, 5 \mathrm{c}$ and $5 \mathrm{~d}$ ). To investigate the tumor-suppressing mechanism underlying epirubicin therapy, HCC samples were subjected to various histological analyses. TUNEL staining revealed a significant increase in the number of apoptotic cells in epirubicin-treated tumors as compared with the control group (Figure 6a). On the other hand, there was a significant reduction of Ki67-positive proliferating cells in the epirubicin-treated HCC (Figure 6b). Interestingly, the number of PECAM-1-positive blood vessels was also decreased in epirubicin-treated HCC (Figure 6c). Therefore, short-term and low-dose epirubicin chemotherapy had perturbed HCC proliferation through apoptosis induction as well as angiogenesis inhibition. Moreover, no significant correlation was obtained between the perfusion data (CT scan) and microvessel density changes (Figure 6c).

\section{Discussion}

For the first time, the current study has demonstrated the feasibility of ultrasound-guided implantation of 


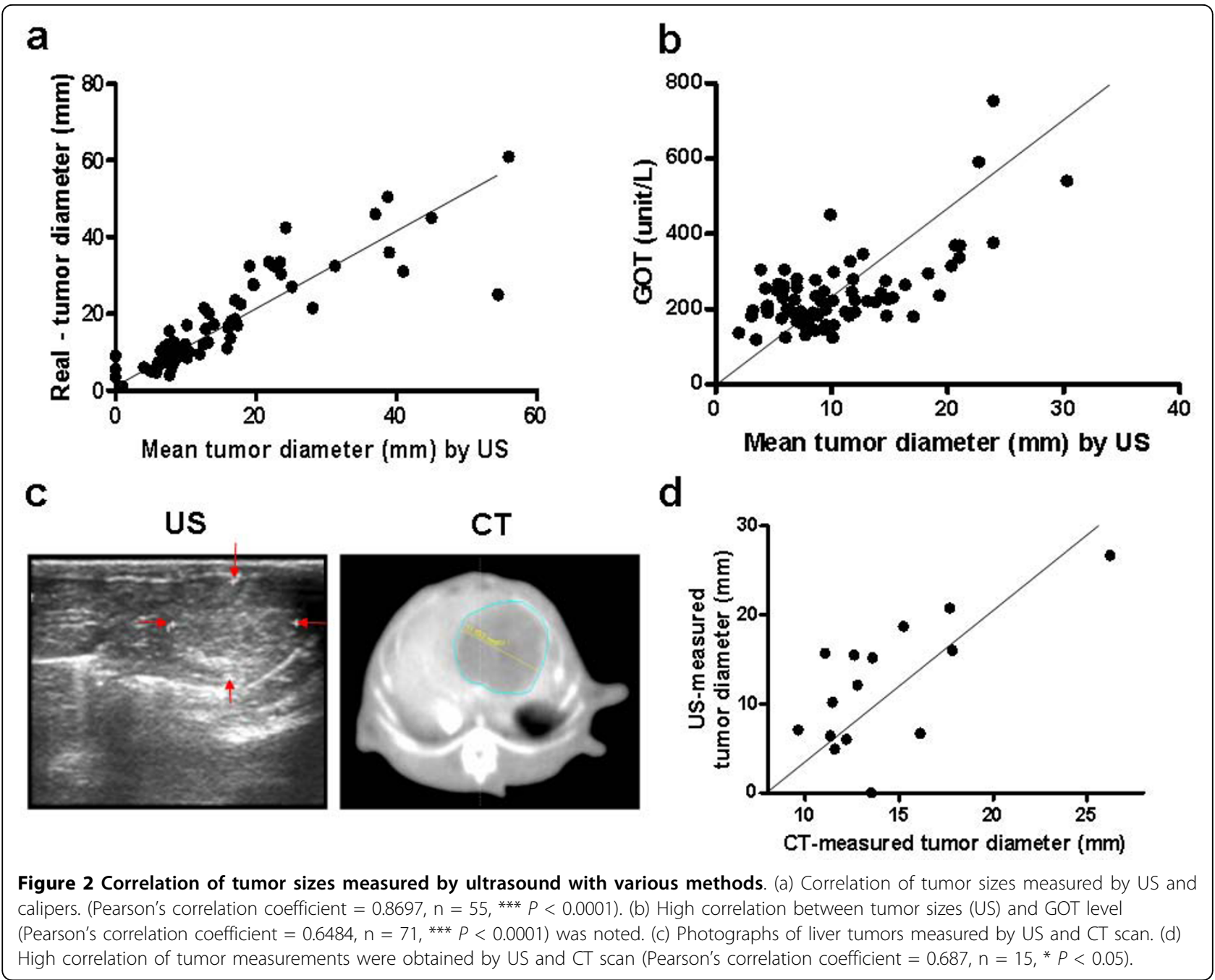

tumor cells into the native liver to create orthotopic $\mathrm{HCC}$ in immune-competent rodent. Compared with other HCC models including laparotomy, it possesses the advantages of easy manipulation as it took less than two hours to accomplish the implantation procedure in twenty animals, minimal trauma to the experimental animals, high reproducibility, early disease onset as reflected in the early detection of tumor by US within 10 days after implantation, and the ability to evaluate the effects of drug therapy on the tumors. Moreover, the results of tumor measurement by US were consistent with those assessed using functional CT scan and the actual sizes measured after sacrificing the animals, suggesting that US is an accurate and reliable means of size assessment of the induced tumors.

There are limited chemotherapeutic options for treatment of advanced HCC. Although the efficacies of many chemotherapeutic agents have been tested in treating $\mathrm{HCC}$, the response rate is disappointing and ranges between $10 \%$ and $15 \%$. Besides, no survival advantage has been demonstrated [21]. This may be due to the unbearable side effects of the conventional maximum tolerated dose (MTD) of chemotherapy upon patients who already have chronic liver diseases and cirrhosis, and the diversity in drug sensitivity due to genetic variations among the cancer cells [22]. In addition, tumor organization further diminishes chemotherapeutic efficacy through the development of multi-cellular drug resistance [23]. On the other hand, the method of metronomic chemotherapy is believed to attack the vascular system of the tumor instead of tumor parenchyma $[14,15]$. It has been demonstrated that this antiangiogenic property can facilitate the cytotoxic effects of chemotherapeutic agents on the cancer cells [24-26]. This regimen can theoretically improve the tolerance of patients and prolong the treatment period, thereby enhancing the therapeutic efficacy of the cytotoxic drugs on the tumor cells.

Evidence from both basal and clinical researches has demonstrated the therapeutic effects of metronomic 

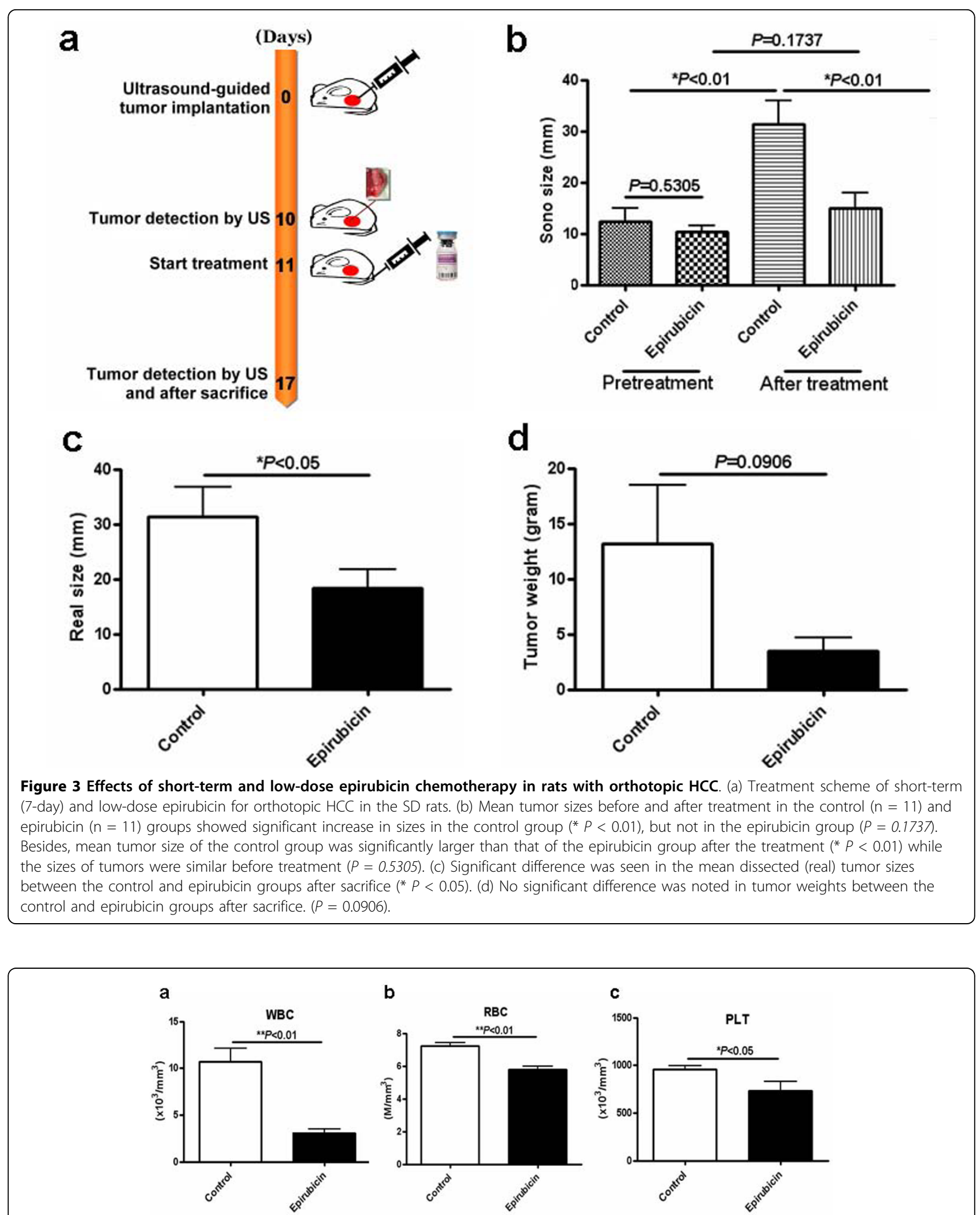

Figure 4 Bone marrow suppression resulting from short-term and low-dose epirubicin chemotherapy was expressed by differences in peripheral blood cells between the two groups. (a) Differences in the WBC (** $P<0.01)$, (b) the RBC $(* P<0.01)$ and (c) the platelet $\left({ }^{*} P<0.05\right)$ counts between the treatment group $(n=11)$ and the control $(n=11)$ group. 


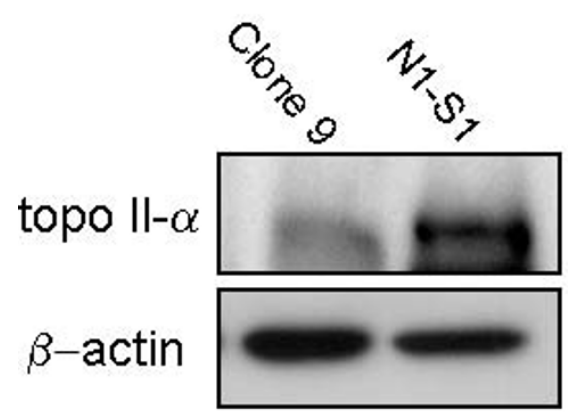

a

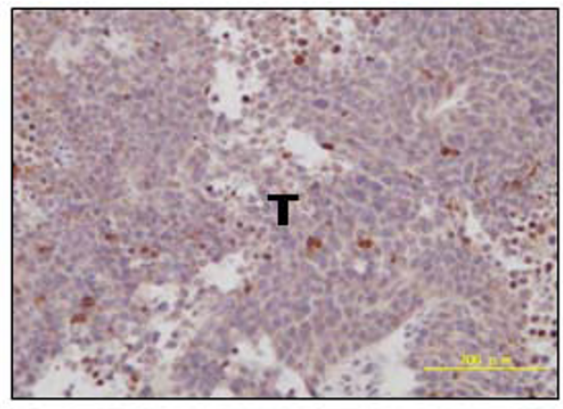

C

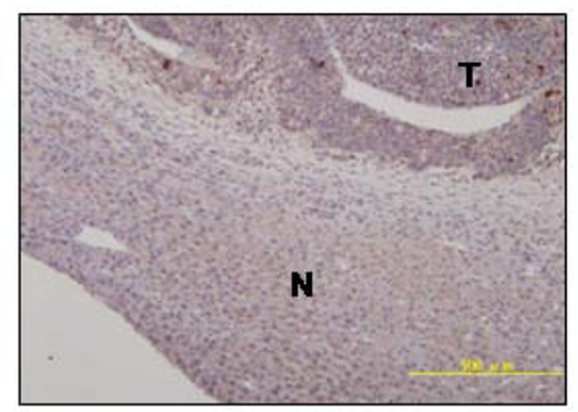

b

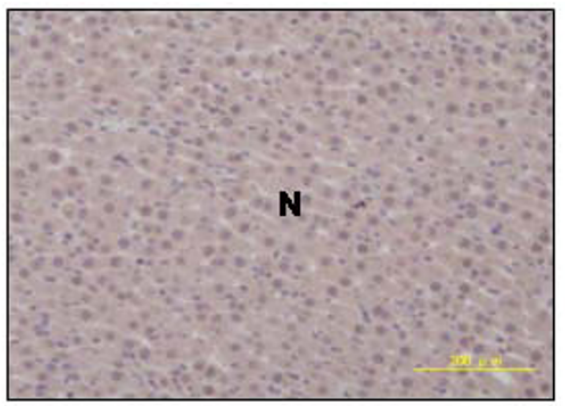

d

Figure 5 Topoisomerase II- $\alpha$ (TOP2A) expression in N1-S1 HCC. (a) Protein extracts isolated from N1-S1 HCC cells and normal hepatocytes (Clone 9) were subjected to the western blot analysis using TOP2A antibodies (1: 1000 dilution). As an internal control, the $\beta$-actin level was also determined. (b) Nuclear and cytoplasm TOP2A expressions in tumor (T) and non-tumor (N) specimens from rat HCC were shown. Original magnification $\times 100$, the insets were then further magnified $(\times 200)$ as shown in $(c)$ tumor and (d) non-tumor parts.

chemotherapy on certain kinds of solid tumors such as ovarian [27] and breast cancers. Colleoni et al. [28] have shown in their clinical trial that low-dose metronomic cyclophosphamide plus methotrexate is effective in the treatment of metastatic breast cancer with cheaper cost and prolonged tolerance to treatment. However, similar reports on the treatment of HCC are rarely found in the literature.

TOP2A over-expression seems to be correlating with the aggressiveness of HCC in terms of early age onset, advanced histological grading, microvascular invasion, chemoresistence, tumor recurrence and mortality $[29,30]$. These facts highlight the potential therapeutic value of epirubicin in targeting TOP2A in the management of HCC. Besides, epirubicin is one of the most frequently used chemotherapeutic agents in the treatment of HCC, either alone or in combination with other cytotoxic drugs, through various routes of administration including TACE (transcatheter chemoembolization), systemic chemotherapy, and trans-catheter arterial infusion chemotherapy [31-37].

Although the present study is only a short-term investigation that fails to elucidate the long-term therapeutic impact of epirubicin on $\mathrm{HCC}$ in an orthotopic animal model, several findings are noteworthy. Firstly, we found over-expression of TOP2A in N1-S1 HCC both in the protein and the histology levels. Secondly, we found a significant effect of the current regimen of epirubicin chemotherapy on HCC growth in this brief animal trial despite the association of bone marrow suppression. Thirdly, the short-term and low-dose epirubicin had high therapeutic potential in terms of histological changes of HCC. It significantly suppressed the proliferation of HCC cells as shown by the Ki-67 staining in comparison with the control group. In addition, TUNEL revealed an increase in the extent of apoptosis of HCC after chemotherapy. Furthermore, and perhaps most importantly, angiogenesis of the tumor endothelium decreased in the treatment group. However, the optimal dosages of metronomic chemotherapy, either chemopreventive or therapeutic, remain elusive [38]. Moreover, our orthotopic HCC animal model was not without shortcomings such as a lack of concurrent chronic liver diseases or cirrhosis that could not completely mimic the clinical condition.

Furthermore, Tang et al., [39] had recently reported an orthotopic advanced HCC model in which significantly improved overall survival was observed using 


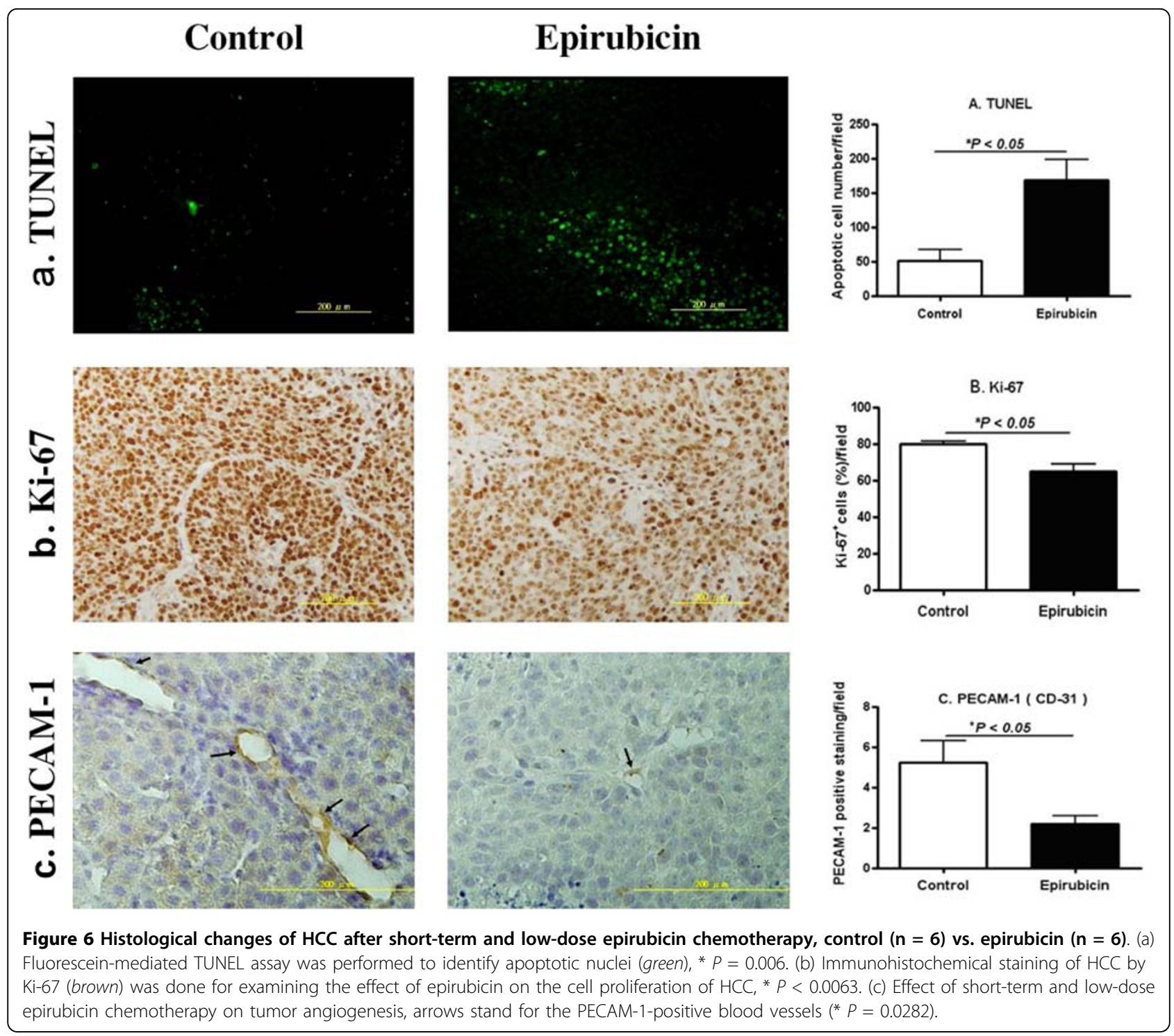

various combinations of metronomic chemotherapy regimens with targeted anti-angiogenic drugs. Through implantation of tumor cells into the native liver through laparotomy, they successfully created orthotopic HCC in SCID mice. The size of the tumor was then monitored by using a novel non-invasive approach (transplantation of tumor cells that have been transfected with the $\beta$-hCG gene). The results of that study are impressive and encouraging. Both that study and our present study have successfully established valuable orthotopic HCC models that can be followed noninvasively either using a gene transfection method or sonographically. Our method has the additional advantage of being close to the clinical situation in which ultrasound is the major tool for following tumor progression. Also, both studies have demonstrated the potential of low-dose chemotherapy, either used alone or in combination with anti-angiogenic agents, in the treatment of HCC.

\section{Conclusions}

In this study, encouraging result has been obtained by the ultrasound-guided tumor implantation of N1-S1 cells that has led to the growth of orthotopic HCC in about $60 \%$ of SD rats, which is comparable to the method of laparotomy. It is a fairly effective and feasible method for establishing an animal model of HCC for future therapeutic trials. It greatly reduces the time period expected for the tumor response to the experimental drugs. Moreover, we have shown the therapeutic efficacies of low-dose epirubicin on HCC growth in terms of tumor size, cancer cell proliferation, apoptosis as well as angiogenesis. On the other hand, the optimal dose of metronomic epirubicin should be substantiated by 
modifications of the current orthotopic animal model and running clinical trials in the future.

\section{Additional material}

Additional file 1: Affidavit of approval of animal use protocol. The animal use protocol 'Development of novel diagnostic and therapeutic strategies for hepatocellular carcinoma' has been reviewed and approved by the institutional animal care and use committee (IACUC) of Kaohsiung Veterans General Hospital.

\section{Acknowledgements}

This study was funded in part by grants from Kaohsiung Veterans General Hospital, Taiwan (VGHKS97G-11, HHC; VGHKS99-022, EMW), the National Science Council, Taiwan (NSC-95-2320-B-075B-003-MY3), Chang Gung Memorial Hospital, Taiwan (CMRPG 880531, CKS), and National Sun Yat-sen University-Kaohsiung Medical University Joint Research Center, Taiwan. The authors thank Miss Yee-Man Chan and Miss Wai-Ching Chan for checking of English grammar.

\section{Author details}

'Department of Biological Sciences, National Sun Yat-sen University, 70 LienHai Road, Kaohsiung 80424, Taiwan. ²Division of Gastroenterology, Department of Internal Medicine, Kaohsiung Veterans General Hospital, 386 Ta-Chung 1st Road, Kaohsiung 81362, Taiwan. ${ }^{3}$ Department of Education and Research, Kaohsiung Veterans General Hospital, 386 Ta-Chung 1st Road, Kaohsiung 81362, Taiwan. ${ }^{4}$ Department of Radiology, Kaohsiung Veterans General Hospital, 386 Ta-Chung 1st Road, Kaohsiung 81362, Taiwan. ${ }^{5}$ Institute of Biomedical Sciences, National Sun Yat-sen University, 70 Lien-Hai Road, Kaohsiung, 80424, Taiwan. 'Division of General Surgery, Department of Surgery, Chang Gung Memorial Hospital - Kaohsiung Medical Center, Chang Gung University College of Medicine, 123 Ta-Pei Road, Niao-Sung Hsiang, Kaohsiung Hsien 83304, Taiwan. ${ }^{7}$ School of Medicine, National Yang-Ming University, No 155, Sec 2, Li-Nong Street, Pei-Tou, Taipei 112, Taiwan. ${ }^{8}$ Division of Hepato-Gastroenterology, Department of Internal Medicine, Chang Gung Memorial Hospital - Kaohsiung Medical Center, Chang Gung University College of Medicine, 123 Ta-Pei Road, Niao-Sung Hsiang, Kaohsiung Hsien 83304, Taiwan.

\section{Authors' contributions}

$\mathrm{HHC}, \mathrm{MHT}$, JTC, HBP and KHL designed the study and analyzed the data. $\mathrm{HHC}, \mathrm{THH}$ and $\mathrm{MHT}$ were responsible for writing the manuscript and revising it critically for important intellectual content. CKS performed the surgery in animals. HHC, THC HFC, EMW and HMK performed the immunoblot, biochemical, pathological and ultrasound experiments. All authors read and approved the final manuscript.

\section{Competing interests}

The authors declare that they have no competing interests.

Received: 14 March 2010 Accepted: 22 July 2010

Published: 22 July 2010

\section{References}

1. El-Serag HB: Hepatocellular carcinoma: an epidemiologic view. J Clin Gastroenterol 2002, 35(5 Suppl 2):S72-S78.

2. Trevisani F, D'Intino PE, Grazi GL, Caraceni P, Gasbarrini A, Colantoni A Stefanini GF, Mazziotti A, Gozzetti G, Gasbarrini G, Bernardi M: Clinical and pathologic features of hepatocellular carcinoma in young and older Italian patients. Cancer 1996, 71(11):2223-2232.

3. Befeler AS, Di-Biscegle AM: Hepatocellular carcinoma: diagnosis and treatment. Gastroenterology 2002, 122(6):1609-1619.

4. Bruix J, Llovet JM: HCC surveillance: who is the target population? Hepatology 2003, 37(3):507-509.

5. Poon D, Anderson BO, Chen LT, Tanaka K, Lau WY, Van Cutsem E, Singh $H$, Chow WC, Ooi LL, Chow P, Khin MW, Koo WH: Management of hepatocellular carcinoma in Asia: consensus statement from the Asian Oncology Summit 2009. Lancet Oncol 2009, 10(11):1111-1118.

6. Di Maio M, De Maio E, Perrone F, Pignata S, Daniele B: Hepatocellular carcinoma: systemic treatments. J Clin Gastroenterol 2002, 35(5 Suppl 2): S109-S114.

7. Armengol C, Tarafa G, Boix L, Solé M, Queralt R, Costa D, Bachs O, Bruix J, Capellá G: Orthotopic Implantation of Human Hepatocellular carcinoma in mice Analysis of tumor progression and establishment of the BCLC-9 cell line. Clin Cancer Res 2004, 10(6):2150-2157.

8. Schmitz V, Tirado-Ledo L, Tiemann K, Raskopf E, Heinicke T, Ziske C, González-Carmona MA, Rabe C, Wernert N, Prieto J, Qian C, Sauerbruch T, Caselmann WH: Establishment of an orthotopic tumor model for hepatocellular carcinoma and non-invasive in vivo tumor imaging by high resolution ultrasound in mice. J Hepatol 2004, 40(5):787-791.

9. Meloni MF, Livraghi T, Filice C, Lazzaroni S, Calliada F, Perretti L: Radiofrequency ablation of liver tumors: the role of microbubble ultrasound contrast agents. Ultrasound Q 2006, 22(1):41-47.

10. Pohl J, Zuna I, Stremmel W, Rudi J: Systemic chemotherapy with epirubicin for treatment of advanced or multifocal hepatocellular carcinoma. Chemotherapy 2001, 47(5):359-365.

11. Zhu AX, Fuchs CS, Clark JW, Muzikansky A, Taylor K, Sheehan S, Tam K, Yung E, Kulke MH, Ryan DP: A phase II study of epirubicin and thalidomide in unresectable or metastatic hepatocellular carcinoma. Oncologist 2005, 10(6):392-398.

12. Hamada A, Yamakado K, Nakatsuka A, Takaki H, Akeboshi M, Takeda K: Hepatic arterial infusion chemotherapy with use of an implanted port system in patients with advanced hepatocellular carcinoma: prognostic factors. J Vasc Interv Radiol 2004, 15(8):835-841.

13. Plosker $G L$, Epirubicin Faulds D: A review of its pharmacodynamic and pharmacokinetic properties, and therapeutic use in cancer chemotherapy. Drugs 1993, 45(5):788-856.

14. Browder T, Butterfield CE, Kräling BM, Shi B, Marshall B, O'Reilly MS, Folkman J: Antiangiogenic scheduling of chemotherapy improves efficacy against experimental drug-resistant cancer. Cancer Res 2000, 60(7):1878-1886.

15. Klement G, Baruchel S, Rak J, Man S, Clark K, Hicklin DJ, Bohlen P, Kerbel RS: Continuous low-dose therapy with vinblastine and VEGF receptor-2 antibody induces sustained tumor regression without overt toxicity. $J$ Clin Invest 2000, 105(8):R15-24.

16. Lam T, Hetherington JW, Greenman J, Maraveyas A: From total empiricism to a rational design of metronomic chemotherapy phase I dosing trials. Anticancer Drugs 2006, 17(2):113-121.

17. Yu J, Qiao L, Zimmermann L, Ebert MP, Zhang H, Lin W, Röcken C, Malfertheiner P, Farrell GC: Troglitazone inhibits tumor growth in hepatocellular carcinoma in vitro and in vivo. Hepatology 2006, 43(1):134-143

18. Kan Z, Phongkitkarun S, Kobayashi S, Tang Y, Ellis LM, Lee TY, Charnsangavej C: Functional CT for quantifying tumor perfusion in antiangiogenic therapy in a rat model. Radiology 2005, 237(1):151-158.

19. Bertazzoli C, Rovero C, Ballerini L, Lux B, Balconi F, Antongiovanni V, Magrini U: Experimental systemic toxicology of 4'-epidoxorubicin, a new, less cardiotoxic anthracycline antitumor agent. Toxicol Appl Pharmacol 1985, 79(3):412-422.

20. Launchbury AP, Habboubi N: Epirubicin and doxorubicin: a comparison of their characteristics, therapeutic activity and toxicity. Cancer Treat Rev 1993, 19(3):197-228.

21. Abou-Alfa GK, Huitzil-Melendez FD, O'Reilly EM, Saltz LB: Current management of advanced hepatocellular carcinoma. Gastrointest Cancer Res 2008, 2(2):64-70.

22. Hahnfeldt $P$, Folkman J, Hlatky L: Minimizing long-term tumor burden: the logic for metronomic chemotherapeutic dosing and its antiangiogenic basis. J Theor Biol 2003, 220(4):545-554.

23. Kerbel RS, Rak J, Kobayashi H, Man MS, StCroix B, Graham CH: Multicellular resistance: a new paradigm to explain aspects of acquired drug resistance of solid tumors. Cold Spring Harb Symp Quant Biol 1994 59:661-672.

24. Jain RK: Delivery of novel therapeutic agents in tumors physiological barriers and strategies. J Natl Cancer Inst 1989, 81(8):570-576.

25. Jain RK: Tumor angiogenesis and accessibility: role of vascular endothelial growth factor. Semin Oncol 2002, 29(6 Suppl 16):3-9. 
26. Gorski DH, Beckett MA, Jaskowiak NT, Calvin DP, Mauceri HJ, Salloum RM, Seetharam S, Koons A, Hari DM, Kufe DW, Weichselbaum RR: Blockage of the vascular endothelial growth factor stress response increases the antitumor effects of ionizing radiation. Cancer Res 1999, 59(14):3374-3378.

27. Kamat AA, Kim TJ, Landen CN Jr, Lu C, Han LY, Lin YG, Merritt WM, Thaker PH, Gershenson DM, Bischoff FZ, Heymach JV, Jaffe RB, Coleman RL, Sood AK: Metronomic chemotherapy enhances the efficacy of antivascular therapy in ovarian cancer. Cancer Res 2007, 67(1):281-288.

28. Colleoni M, Orlando L, Sanna G, Rocca A, Maisonneuve P, Peruzzotti G Ghisini R, Sandri MT, Zorzino L, Nolè F, Viale G, Goldhirsch A: Metronomic low-dose oral cyclophosphamide and methotrexate plus or minus thalidomide in metastatic breast cancer: antitumor activity and biological effects. Ann Oncol 2006, 17(2):232-238.

29. Watanuki A, Ohwada S, Fukusato T, Makita F, Yamada T, Kikuchi A Morishita Y: Prognostic significance of DNA topoisomerase II alpha expression in human hepatocellular carcinoma. Anticancer Res 2002, 22(2B):1113-1119.

30. Wong N, Yeo W, Wong WL, Wong NL, Chan KY, Mo FK, Koh J, Chan SL, Chan AT, Lai PB, Ching AK, Tong JH, Ng HK, Johnson PJ, To KF: TOP2A overexpression in hepatocellular carcinoma correlates with early age onset, shorter patients survival and chemoresistance. Int J Cancer 2009, 124(3):644-652.

31. Edeline J, Raoul JL, Vauleon E, Guillygomac'h A, Boudjema K, Boucher E: Systemic chemotherapy for hepatocellular carcinoma in non-cirrhotic liver: A retrospective study. World J Gastroenterol 2009, 15(6):713-716.

32. Takamatsu M, Matsuda T, Kawaguchi K, Ku Y: A complete response to oneshot hepatic arterial infusion of epirubicin in a patient with highly advanced hepatocellular carcinoma. Gan To Kagaku Ryoho 2007, 34(12):2102-2104.

33. Luo JJ, Yan ZP, Wang JH, Liu QX, Chen Y: Transhepatic arterial chemoembolization by epirubicin mixed with microspheres for hepatocellular carcinoma. Zhonghua Zhong Liu Za Zhi 2007, 29(8):619-622.

34. Ishikawa T, Imai M, Kamimura H, Tsuchiya A, Togashi T, Watanabe K, Seki K, Ohta H, Yoshida T, Kamimura T: Improved survival for hepatocellular carcinoma with portal vein tumor thrombosis treated by intra-arterial chemotherapy combining etoposide, carboplatin, epirubicin and pharmacokinetic modulating chemotherapy by 5-FU and enteric-coated tegafur/uracil: a pilot study. World J Gastroenterol 2007, 13(41):5465-5470.

35. Tanaka T, Ikeda M, Okusaka T, Ueno H, Morizane C, Ogura T, Hagihara A, Iwasa S: A phase II trial of transcatheter arterial infusion chemotherapy with an epirubicin-Lipiodol emulsion for advanced hepatocellular carcinoma refractory to transcatheter arterial embolization. Cancer Chemother Pharmacol 2008, 61(4):683-688.

36. Yamashita S, Niinobu T, Nakagawa S, Amano M, Nishikawa Y, Hayashida H, Higaki N, Fujita M, Sakon M: Hepatic arterial infusion chemotherapy for advanced hepatocellular carcinoma providing a good QOL. Gan To Kagaku Ryoho 2006, 33(12):1928-1930.

37. Sergio A, Cristofori C, Cardin R, Pivetta G, Ragazzi R, Baldan A, Girardi L, Cillo U, Burra P, Giacomin A, Farinati F: Transcatheter arterial chemoembolization (TACE) in hepatocellular carcinoma (HCC): the role of angiogenesis and invasiveness. Am J Gastroenterol 2008, 103(4):914-921.

38. Satti J: The emerging low-dose therapy for advanced cancers. Dose Response 2009, 7(3):208-220.

39. Tang TC, Man S, Lee CR, Xu P, Kerbel RS: Impact of metronomic UFT/ Cyclophosphamide chemotherapy and antiangiogenic drug assessed in a new preclinical model of locally advanced orthotopic hepatocellular carcinoma. Neoplasia 2010, 12(3):264-274.

\section{Pre-publication history}

The pre-publication history for this paper can be accessed here: http://www.biomedcentral.com/1471-230X/10/83/prepub

doi:10.1186/1471-230X-10-83

Cite this article as: Chan et al:: Rapid induction of orthotopic

hepatocellular carcinoma in immune-competent rats by non-invasive ultrasound-guided cells implantation. BMC Gastroenterology 2010 10:83.

\section{Submit your next manuscript to BioMed Central and take full advantage of:}

- Convenient online submission

- Thorough peer review

- No space constraints or color figure charges

- Immediate publication on acceptance

- Inclusion in PubMed, CAS, Scopus and Google Scholar

- Research which is freely available for redistribution

Submit your manuscript at www.biomedcentral.com/submit 